\title{
Atomic force microscopic investigation of commercial pressure sensitive adhesives for forensic analysis
}

\author{
Elisabetta Canetta* and Ashok K. Adya ${ }^{\dagger}$ \\ Condensed Matter Group and BIONTHE Centre, Division of Biotechnology and Forensic \\ Sciences, School of Contemporary Sciences, University of Abertay Dundee, \\ Bell Street, Dundee DDI 1HG, Scotland, UK
}

\begin{abstract}
Pressure sensitive adhesives (PSAs), such as those used in packaging and adhesive tapes, are very often encountered in forensic investigations. In criminal activities, packaging tapes may be used for sealing packets containing drugs, explosive devices, or questioned documents, while adhesive and electrical tapes are used occasionally in kidnapping cases. In this work, the potential of using Atomic Force Microscopy (AFM) in both imaging and force mapping (FM) modes to derive additional analytical information from PSAs is demonstrated. AFM has been used to illustrate differences in the ultrastructural and nanomechanical properties of three visually distinguishable commercial PSAs to first test the feasibility of using this technique. Subsequently, AFM was used to detect nanoscopic differences between three visually indistinguishable PSAs.
\end{abstract}

Keywords: Atomic force microscopy (AFM); Force mapping; Nanotechnology; Pressure sensitive adhesives (PSA), Mechanical properties, Items of recovery

\footnotetext{
* Present address: School of Physics and Astronomy, University of St Andrews, North Haugh, St Andrews, Fife, KY16 9SS, Scotland, UK

† Corresponding author: a.k.adya@abertay.ac.uk, Tel.: +44-(0)-1382-308653, Fax: +44-(0)-1382-308663

₹ Bio- and Nano-Technologies for Health and Environment
} 


\section{Introduction}

A reliable characterisation of the items of recovery from a crime scene requires the most accurate forensic analyses using the state-of-the art techniques [1]. Atomic force microscopy [2] has recently emerged as a possible useful tool for surface characterisation of a variety of materials through the analysis of their morphology and mechanical properties. Amongst various other materials, such as hair [3], body fluids [4], documents [5], textile fibres [6, 7], and polymers [8], pressure sensitive adhesives (PSAs) [9-11] are also encountered in forensic investigations.

Pressure sensitive adhesive tapes are employed in a variety of criminal activities such as the restraint of an individual during robbery and offences against a victim, the concealment and packaging of controlled drugs, and the enclosure of explosive devices. Packaging materials including PSAs are produced in vast amounts and are ubiquitous in our modern society. It is broadly acknowledged that routinely used techniques (e.g. Fourier transform infrared spectroscopy, FT-IR, and pyrolisis-gaschromatography-mass-spectrometry, Py-GC-MS) in forensic science laboratories to identify chemical constituents [12-16] for discriminating PSAs are mostly effective $[17,18]$. However, AFM can provide supplementary and useful analytical data on PSAs due to its capability to map the surface morphological and mechanical properties of the adhesives. Also, since PSA technology is based on finding the right balance between the cohesive and viscous properties of a polymer, their properties vary on different length scales ranging from macro- to nano- scales, and AFM can render the nanoscopic information. The value of a forensic analysis lies in its ability to interpret the physical data obtained from items of recovery found at a crime scene and, hopefully, to link a particular suspect/source to it. The Scientific Working Group for Materials Analysis (SWGMAT) of the Federal Bureau of Investigation (FBI) have 
reported the standards and guidelines for the forensic examination of pressuresensitive tapes [19].

In this work, the potential of using AFM in the imaging mode to examine the morphology of the specimens, as well as in the force mapping (FM) mode to investigate their mechanical properties to characterise PSAs is demonstrated. The ability of AFM to observe the nanostructural and nanomechanical differences of commercial PSAs was first tested by analysing three visually distinguishable adhesive tapes viz. transparent OPP packaging tapes, brown packaging tapes, and green electrical insulation tapes. Subsequently, colourless and transparent OPP packaging tapes from three UK distributors were analysed by AFM to verify the capability of this nanotool to show the finest surface differences of similar PSAs that are visually indistinguishable.

\section{Experimental}

\subsection{Commercial pressure sensitive adhesives}

Three different common commercial adhesives viz. transparent cello tape made of regenerated cellulose, brown packaging tape made of a waterproof low-static polypropylene film, and green electrical insulation tape made of a PVC (polyvinylchloride) film (Advanced Tapes International Limited, Leicester, England) were investigated. These were followed by the investigation of colourless and transparent OPP packaging tapes from three UK distributors, namely Niceday - Large core office, Henkel - Adhesion J1626, and Eureka - Large clear tape.

\subsection{Atomic force microscopy (AFM) imaging}

The ultrastructure of the commercial PSAs was determined by using a commercial NTEGRA - AFM (NT-MDT, Moscow, Russia). The surface of each adhesive was scanned in semi-contact mode. $1 \mathrm{~cm}^{2}$ of each adhesive was mounted on 
a microscope glass slide by using a double-sided tape ensuring that the adhesive was levelled and strongly adhered to the slide.

The surface topography of each adhesive was imaged by using silicon cantilevers (ATEC-NC, Nanosensors, Switzerland) with a nominal spring constant of $45 \mathrm{~N} / \mathrm{m}$ and a high resonance frequency $(330 \mathrm{kHz})$. The AFM images $(5 \mu \mathrm{m} \times 5 \mu \mathrm{m}$ in size and $512 \times 512$ pixels) were collected using a scan speed of $1.56 \mathrm{~Hz}$. A lateral resolution of $30 \mathrm{~nm}$ was estimated for all the captured images.

The imaging experiments were repeated on three randomly chosen areas of each slide and five samples were scanned, thereby giving a total of 15 measurements on each adhesive. Apart from the visual observation of the surface morphology of each adhesive which was found to be consistent between different measurements, surface roughness analysis performed on each image clearly confirmed the repeatability and reliability of the AFM measurements. The roughness of the adhesive surfaces was analysed by measuring the root mean square roughness, $R_{r m s}$, on the whole AFM height image. $\mathrm{R}_{\mathrm{rms}}$ is defined as the standard deviation from the mean data plane of the $h$ (height) values of the AFM images within a selected region on the surface:

$$
R_{r m s}=\sqrt{\frac{\sum_{i=1}^{N}\left(h_{i}-\bar{h}\right)^{2}}{N}},
$$

where $h_{i}$ is the current height value, $\bar{h}$, the height of the mean data plane, and $N$, the number of points within the selected region of a given area. This analysis was carried out on raw AFM images (i.e. images that were neither flattened nor elaborated with any filter) by using the NT-MDT software. The results are reported in Table 1 as average surface roughness \pm standard deviation.

The amplitude of the oscillation when the tip was in contact with the PSA surface was kept just below the free amplitude, which was typically $295 \mathrm{~nm}$, in order to image the PSA surface in "soft-tapping" conditions to minimize indentation [8, 
20,21] and damage to the surface. A simultaneously generated phase image provided information on the energy dissipation between the tip and the sample surface $[8,22]$. Dark regions in the phase images represented greater energy dissipation between the tip and cantilever. The greater viscous component of the viscoelasticity [23] is responsible for causing this dissipation of energy. Brighter regions were attributed to a surface with a lower viscous component and greater elasticity.

\subsection{AFM force mapping (AFM-FM)}

The nanomechanical properties of each of the commercial PSA were investigated by using the same AFM in force spectroscopy (AFM-FS) mode in order to create property maps of adhesive force and adhesion energy of the PSAs [8]. The AFM cantilevers (CSG10, NT-MDT, Moscow, Russia) used in the FS experiments were made from $\mathrm{Si}_{3} \mathrm{~N}_{4}$. These cantilevers were very soft with a nominal spring constant $\sim 0.14 \mathrm{~N} / \mathrm{m}$ and a resonant frequency $\sim 20 \mathrm{kHz}$. The tip (radius of curvature $<10 \mathrm{~nm}$ ) microfabricated on the AFM cantilever was brought into contact with the surface of the adhesive tape. The AFM-FM experiments were carried out over a grid of $20 \times 20$ points on a $5 \times 5 \mu \mathrm{m}^{2}$ area of the adhesive, and the FS curve on each point was recorded. These measurements were again repeated on three randomly chosen areas of each slide and five samples, thus giving a total of 15 measurements for each adhesive. During the AFM-FM experiments, an average normal force of $2 \mathrm{nN}$ was applied by the AFM cantilever onto the adhesive tape. The sample was lowered away from the tip at a speed of $0.45 \mu \mathrm{m} / \mathrm{sec}$. In each experiment, the maximum adhesive force of the particles forming the film to the tip $\left(F_{\max }\right)$, the maximum distance of deformation of these particles $\left(d_{\max }\right)$, and the adhesion energy $(\gamma)$ were measured (Fig. 1). All AFM-FM experiments were performed in contact mode in air at room temperature. 
The FS curves obtained during an AFM-FM experiment were in fact forceheight curves. To transform the force-height curves into force-distance $(F-d)$ curves, the real distance, $d$, between the sample and the AFM tip was calculated by subtracting the deflection of the cantilever, $z$, from the height values that corresponded to the measured piezo displacement, $z_{\text {piezo }}$ :

$$
d=z_{\text {piezo }}-z
$$

Force spectroscopy experiments on adhesive films [24-28] have previously been performed to determine their nanomechanical properties. However, in the AFM-FM experiments presented in this work the surface of the adhesive film was mapped point-by-point and $400 \mathrm{FS}$ curves were collected in each experiment repeated over 15 areas for each adhesive that allowed us to collect statistically significant data [8].

\subsection{Nanomechanical properties}

A commercial software (Gigasoft ProEssentials v3 Package) was used to display the $F$ - $d$ curves. Each of the 400 curves in a data set was individually shifted to remove its offset. The maximum adhesive force of the tip to surface $\left(F_{\max } / \mathrm{nN}\right)$, the maximum distance of deformation of the sample $\left(d_{\max } / \mu \mathrm{m}\right)$, and the adhesion energy $\left(\gamma / 10^{-15} \mathrm{~J}\right)$ were calculated from the $F-d$ curves by using a dedicated program written with Borland Delphi 7.0 software. The adhesion energy, $\gamma$, was obtained by integrating the area under the $F$ - $d$ curve.

\section{Results and discussion}

\subsection{Nanostructure of three visually distinguishable PSAs}

To test the ability of AFM to visualise the finest differences between PSAs, three visually distinguishable commercial adhesive tapes were studied. The AFM phase images (size $5 \times 5 \mu^{2}$ ) of these tapes are depicted in Fig. 2. Portions of these images zoomed to $2 \times 2 \mu \mathrm{m}^{2}$ are also shown. The images for all the tapes show the 
existence of two phases - a hard phase which is less energy dissipative and a soft phase that is more energy dissipative. It may be pointed out here that if the regions of lower and higher viscosity are dispersed in the adhesive tape or film, the interaction of the AFM tip with the film surface can vary. For instance, when the tip comes in contact with the more viscous region on the surface, more energy gets dissipated and the area being scanned appears dark in colour in the phase image [9]. Due to similar reasoning, less energy is dissipated on a lower viscosity region on the surface and it appears brighter in colour in the phase image [10]. It is thus clear that the phase image can render important information about the viscous properties of different constituent components of the PSA, as well as their distribution and relative abundance on the adhesive film surface. The distribution of the two (hard and soft) phases in each of the three investigated tapes is found to be different. The morphology of the transparent cello tape (Fig. 2a) shows the presence of individual adhesive polymer particles (dark spots) and possibly surfactant molecules (bright spots). In the brown packaging tape (Fig. 2b) hardly any individual polymer particles are discernible, indicating that the particles may have coalesced in this adhesive film. The bright spots in Fig. $2 \mathrm{~b}$ are probably the surfactant molecules. The topography of the electrical insulation tape (Fig. 2c) is entirely different from the other two tapes, and a comparison reveals that the harder phase (bright spots) in it is present in relatively very small amounts.

The morphological differences observed in the AFM images were confirmed quantitatively by surface roughness analysis (Table 1). The brown packaging tape was found to be smoother, $R_{\mathrm{rms}}=(35.3 \pm 2.6) \mathrm{nm}$, while the insulation tape turned out to be rougher, $R_{\mathrm{rms}}=(96.7 \pm 3.8) \mathrm{nm}$, possibly due to the presence of "crevices" on the tape surface. These results prove the capability of AFM to image the finest topological features of visually different PSAs. 
The AFM phase images (size $5 \times 5 \mu^{2}$ ) of three colourless and transparent OPP adhesive tapes are shown in Fig. 3. Portions of these images zoomed to $2 \times 2$ $\mu \mathrm{m}^{2}$ are also shown. On the surface of the Henkel transparent cello tape individual adhesive polymer particles (dark spots) are visible with only a few very small hard particles (bright spots), possibly surfactant molecules, present (Fig. 3a) with a homogeneous distribution. This makes the surface of the Henkel tape quite smooth as confirmed by the roughness analysis $\left(\mathrm{R}_{\mathrm{rms}}=(23.0 \pm 3.8) \mathrm{nm}\right.$, see Table 1$)$.

On the contrary, the surface of the Niceday transparent cello tape shows two distinct hard and soft phases (Fig. 3b). Only some individual polymer particles are visible (dark spots in $2 \times 2 \mu \mathrm{m}^{2}$ image) with most of them having coalesced in this more dissipative (soft) phase. The less dissipative (hard) phase probably constitutes of surfactant particles (bright spots). The presence of two distinct phases makes the surface of this tape rougher $\left(\mathrm{R}_{\mathrm{rms}}=(38.8 \pm 2.1) \mathrm{nm}\right.$, see Table 1$)$ as compared to the Henkel tape.

Finally, the Eureka transparent cello tape showed a reasonably uniform morphology (Fig. 3c). Some polymer particles (dark spots) are still visible but most of them seem to have coalesced making the surface to appear relatively uniform with a randomly distributed hard (bright spots) phase present. The more uniform nature of the adhesive surface is also shown by a decrease in the surface roughness $\left(R_{\mathrm{rms}}=\right.$ $(32.2 \pm 2.7) \mathrm{nm}$ ) as compared to that of the Niceday transparent cello tape (see Table $1)$.

These results demonstrate the ability of AFM to image the finest morphological differences of even otherwise visually indistinguishable PSAs. 
Figure 4 shows the $F$ - $d$ curves obtained on three visually distinguishable tapes. The transparent cello tape depicts two well distinguished set of $F-d$ curves as shown in Fig. 4a. The curve on the left encompasses 3 adhesion peaks and shows a very large deformation, indicating that polymer fibrillation probably occurs during the unloading cycle of the AFM-FM experiment. This behaviour indicates the presence of a very soft and highly viscous phase (possibly the adhesive polymer). The $F$ - $d$ curve on the right (Fig. 4a) shows only 1 very sharp and narrow adhesion peak, corresponding to a very hard phase probably constituting of surfactant molecules. These results endorse the previous observations (see Section 3.1) from, the AFM phase images (Fig. 2a) where two distinct phases (hard and soft) were clearly visible. The brown packaging tape (Fig. 4b) also shows two different sets of $F$ - $d$ curves. On the left, a typical $F$ - $d$ curve with 2 adhesion peaks and a broad deformation is presented, indicating the presence of quite a soft and viscous phase. However, the height of the first adhesion peak $\left(F_{\max }=120 \mathrm{nN}\right)$ is lower than that of the first adhesion peak for the transparent tape $\left(F_{\max }=148 \mathrm{nN}\right)$, see Fig. 4a (Left). Moreover, the deformation of the brown tape $\left(d_{\max }=6.2 \mu \mathrm{m}\right)$ observed in Fig. $4 \mathrm{~b}$ is smaller than the deformation $\left(d_{\max }=8.7 \mu \mathrm{m}\right)$ seen in Fig. $4 \mathrm{a}$ for the transparent tape. These results indicate that although both the transparent and brown tapes are made of highly soft and viscous adhesive polymers, the transparent tape is more viscous and softer than the brown tape, suggesting a higher degree of tackiness of the transparent tape compared to the brown tape. The $F-d$ curve on the right of Fig. $4 \mathrm{~b}$ shows only 1 adhesion peak corresponding to the hard phase of the brown tape. However, the height and width $\left(F_{\max }=75 \mathrm{nN} ; d_{\max }=3.8 \mu \mathrm{m}\right)$ of this peak are higher than those observed in Fig. 4a (Right) for the hard phase of the transparent tape $\left(F_{\max }=55 \mathrm{nN}\right.$; $\left.d_{\max }=1.1 \mu \mathrm{m}\right)$, indicating that the hard phase of the brown tape could be a surfactantrich phase in a mixture rather than pure surfactant as observed for the transparent 
tape. The electrical insulation tape (Fig. 4c) shows a similar behaviour to that of the transparent tape (Fig. 4a) with a very soft and viscous phase (Left, Fig. 4c) and a harder phase (Right, Fig. 4c). However, the soft phase of the electrical insulation tape seems to be harder than that of the transparent cello tape as shown by the smaller heights of the peaks in Fig. 4c (Left) as compared to those in Fig. 4a (Left). Comparison of the $F-d$ curves of the hard phases for the electrical insulation, transparent and brown tapes indicates that the insulation tape has a much softer hard phase than that of both the transparent and brown tapes. This is suggested by the height of the single adhesion peak observed in the electrical insulation tape being much higher $\left(F_{\max }=130 \mathrm{nN}\right)$ than those measured for the hard phases of the transparent and brown tapes (see Fig. 4). This seems to imply that in the insulation tape the hard phase mainly results from an enrichment of the adhesive polymer with the surfactant rather than from pure surfactant alone which seems to be the case for the transparent tape. These results are in agreement with the AFM phase images (see Section 3.1 and Fig. 2c) that show a relatively uniform topography with just a small amount of surfactant present in the electrical insulation tape.

The $F_{\max }, d_{\max }$, and $\gamma$ distributions over $400 F$ - $d$ curves obtained from AFMFM experiments are shown in Fig. 5. Two distinct populations can clearly be observed in all the three distributions for the three investigated tapes. Undoubtedly, these correspond to the two distinct phases revealed earlier by the AFM phase images in Fig. 2 and the $F-d$ curves in Fig. 4. Certain differences are nevertheless discernible. Unlike the transparent cello tape in Fig. 5a, for instance, the brown and electrical insulation packaging tapes in Figs. $5 \mathrm{~b}$ and $5 \mathrm{c}$, respectively show two subpopulations, in particular for the softer phase. This seems to stem from the variation in the nature and composition of the polymer, copolymer, acrylic, surfactant, and tackifier in different types of adhesive tapes that differ in their detailed formulation. It is known for example, that tackifiers are usually added to acrylic 
formulations or polymers to increase the adhesive strength and energy, thereby boosting the peeling force and performance of the PSAs. Poor miscibility can often lead to heterogeneity, thereby resulting in phase separation. Different approaches are commonly used in the formulation processes, such as polymerisation, copolymerisation and blending, and such processes are continually being developed in order to improve the performance of the PSAs for particular applications in mind.

The 2D AFM-FM adhesion energy maps, constructed on a grid of $20 \times 20$ points over a $5 \times 5 \mu \mathrm{m}^{2}$ area of the adhesive tape, shown in Fig. 6, are also found to be different for the three investigated tapes. The adhesion energy map allows visualisation of the precise localisation of different components of the adhesive. Each square in the $2 \mathrm{D}$ map corresponds to one of the 400 points of the AFM-FM experiments and the grey scale is indicative of the magnitude of adhesion energy. The brighter (darker) the square in the grid, the higher (lower) the adhesion energy, and therefore the softer (harder) that particular component of the adhesive. As also shown by the AFM images (see Fig. 2), the distribution of the hard phase (darker squares) is less uniform in the transparent tape (Fig. 6a) in comparison to the brown tape (Fig. 6b). In the transparent tape the hard and soft phases are reasonably well separated with a high contrast between them. The energy map for the electrical insulation tape (Fig. 6c) shows greater homogeneity compared to the map for the transparent tape, and this observation is consistent with the information obtained from the AFM images (see Fig.2).

The above results clearly demonstrate that the morphology of the PSAs revealed by AFM imaging, the AFM $F-d$ curves obtained from AFM force mapping (FM) experiments, the statistical distributions of $F_{\max }, d_{\max }$, and $\gamma$ over $400 F-d$ curves, and the 2D AFM-FM adhesion energy maps are all different for visually distinguishable commercially available adhesive tapes. 
PSAs

The nanomechanical features of three visually similar OPP transparent cello tapes investigated by AFM-FM are shown in Fig. 7. The transparent cello tape by Niceday shows two different sets of $F-d$ curves (Fig. 7a). In the curve on the left, the presence of three adhesion peaks confirms a very large deformation of the adhesive polymer. This could be due to polymer fibrillation happening during the unloading cycle of the AFM-FM experiment. This behaviour is possibly caused by the presence of a very soft and highly viscous phase (adhesive polymer). The $F-d$ curve on the right of Fig. 7a shows only 1 very sharp and narrow adhesion peak, which corresponds to a very hard phase, possibly a surfactant. These results agree with the AFM phase images (see Fig. 3a) where two phases (hard and soft) were clearly visible.

Unlike the cello tape by Niceday, the OPP transparent cello tape by Henkel (Fig. 7b) shows only one set of $F-d$ curves. This confirms the smooth surface of the PSA observed in the AFM images (Fig. 3b). Fig. 7b depicts a typical $F$ - $d$ curve with 2 adhesion peaks, a very large peak and a quite small peak. This broad deformation could be caused by the presence of quite a soft and viscous phase. Interestingly, the height of the first adhesion peak $\left(F_{\max }=88 \mathrm{nN}\right)$ is lower than that of the first adhesion peak for OPP tape by Niceday $\left(F_{\max }=135 \mathrm{nN}\right.$, see the left $F$ - $d$ curve of Fig. 7a). In addition, the deformation of the OPP tape by Henkel $\left(d_{\max }=6.6 \mu \mathrm{m}\right)$ is smaller than the deformation observed in the left $F-d$ curve of Fig. 7a for the transparent tape by Niceday $\left(d_{\max }=9.0 \mu \mathrm{m}\right)$. These findings seem to indicate that although both of the three transparent tapes are made of highly soft and viscous adhesive polymers, the OPP tape by Niceday is more viscous and softer than that by Henkel. This different behaviour could be due to a higher degree of tackiness of the OPP tape by Niceday compared to the OPP tape by Henkel. 
The OPP cello tape by Eureka shows two populations which, however, do not differ much from each other, both showing 1 adhesion peak. The height of the adhesion peak is slightly different for each population $\left(F_{\max }=91 \mathrm{nN}\right.$ for the $F$ - $d$ curve on the left and $F_{\max }=80 \mathrm{nN}$ for the $F$ - $d$ curve on the right), indicating the presence of two phases (soft and hard). The fact that these two populations appear similar could be due to coalescence of the hard and soft phase as also shown earlier by the AFM images (Fig. 3c).

Comparison of the $F$ - $d$ curves of the soft phases for the three OPP transparent, tapes indicates that the soft phase of the Niceday tape is softer than that of the Henkel and Eureka tapes.

The $F_{\max }, d_{\max }$, and $\gamma$ distributions over $400 F$ - $d$ curves obtained from AFMFM experiments are shown in Fig. 8. Two well separated populations can be seen in the $F_{\max }$ and $\gamma$ distributions for the Niceday tape (Fig. 8a). These distributions correspond to the two distinct phases observed in the AFM phase images in Fig. $3 \mathrm{~b}$ and the $F$ - $d$ curves in Fig. 7a. The Henkel tape shows only one distribution for $F_{\max }$ and $d_{\max }$ which agrees with both the AFM images (Fig. 3a) and the $F-d$ curves (Fig. 7b). Surprisingly, $\gamma$ distribution in Fig. $8 \mathrm{~b}$ shows the presence of a softer phase probably due to the presence of two different components (one soft and the other harder) in the adhesive formulation that were not visible in the AFM phase image. The Eureka tape shows the presence of two phases (one soft and the other hard) in all the three distributions, indicating the presence of two components in the formulation of the adhesive as also shown by the AFM images (Fig. 3c). The adhesion peak of softer phase dominates the statistical distributions for $\mathrm{F}_{\max }$ and $\gamma$ which could be due to the coalescence of the hard phase with the soft phase as also seen earlier in the AFM images (Fig. 3c).

The 2D AFM-FM adhesion energy maps, constructed on a grid of $20 \times 20$ points over a $5 \times 5 \mu^{2}$ area of the OPP transparent cello tapes (Fig. 9), are also 
different for the three investigated tapes. As also shown by the AFM images (see Fig. 3), the distribution of the hard phase (darker squares) is quite uniform in the OPP transparent tape by Niceday (Fig. 9a). The hard and soft phases are very well separated with a high contrast between them. The energy map for the OPP tape by Henkel (Fig. 9b) shows greater homogeneity compared to the map for the Niceday tape, and this observation is consistent with the information obtained from the AFM images shown in Fig. 3. Although the energy map for the Eureka tape looks quite uniform (Fig. 9c), it is possible to see the presence of two different components (hard phase corresponding to darker squares and soft phase corresponding to brighter squares) as also confirmed by the AFM images (Fig. 3) and the $F$ - $d$ curves (Fig. 7).

The above findings shed light on the potential of AFM as an additional technique to investigate the finest morphological and nanomechanical differences of visually indistinguishable PSAs.

\section{Conclusions}

The present results have shown for the first time that nanotechnology techniques, such as AFM imaging and AFM force mapping (AFM-FM) can be employed to obtain useful additional analytical information from pressure sensitive adhesives commonly found in forensic examination. These AFM techniques have been used successfully to demonstrate differences in ultrastructural and nanomechanical properties of different pressure sensitive adhesive (PSA) tapes. Statistical distributions of adhesion force $\left(F_{\max }\right)$ and adhesion energy $(\gamma)$ clearly showed the existence of distinct phases in PSAs whose distributions varied from one adhesive to another. AFM techniques can thus provide supplementary data at the nanoscale in the forensic examination of adhesives. 


\section{Acknowledgements}

The authors wish to acknowledge the University of Abertay Dundee (UAD) for the support of this work. 


\section{Tables}

Surface Roughness (nm)

\begin{tabular}{|c|c|c|c|c|c|c|}
\hline Adhesive tape & $\begin{array}{c}\text { Sample } \\
1^{*}\end{array}$ & $\begin{array}{c}\text { Sample } \\
2^{*}\end{array}$ & $\begin{array}{c}\text { Sample } \\
3^{*}\end{array}$ & $\begin{array}{c}\text { Sample } \\
4^{*}\end{array}$ & $\begin{array}{c}\text { Sample } \\
5^{*}\end{array}$ & Average $^{\dagger}$ \\
\hline $\begin{array}{l}\text { Transparent } \\
\text { cello tape }\end{array}$ & $40.2 \pm 3.5$ & $44.7 \pm 3.2$ & $43.5 \pm 1.0$ & $39.1 \pm 1.3$ & $42.4 \pm 2.7$ & $42.0 \pm 3.0$ \\
\hline $\begin{array}{c}\text { Brown } \\
\text { packaging }\end{array}$ & $33.2 \pm 1.7$ & $32.8 \pm 3.9$ & $34.0 \pm 3.4$ & $34.7 \pm 2.5$ & $34.0 \pm 3.0$ & $35.3 \pm 2.6$ \\
\hline $\begin{array}{l}\text { Green electrical } \\
\text { insulation }\end{array}$ & $99.0 \pm 3.2$ & $95.2 \pm 3.4$ & $93.7 \pm 3.7$ & $95.7 \pm 3.8$ & $99.7 \pm 3.9$ & $96.7 \pm 3.8$ \\
\hline $\begin{array}{c}\text { Transparent OPP } \\
\text { (Henkel) }\end{array}$ & $17.9 \pm 4.6$ & $24.1 \pm 1.0$ & $26.4 \pm 2.5$ & $22.4 \pm 1.8$ & $24.3 \pm 3.1$ & $23.0 \pm 3.8$ \\
\hline $\begin{array}{c}\text { Transparent OPP } \\
\text { (Niceday) }\end{array}$ & $41.0 \pm 3.9$ & $36.7 \pm 1.7$ & $41.1 \pm 2.8$ & $36.9 \pm 2.9$ & $38.4 \pm 2.8$ & $38.8 \pm 2.1$ \\
\hline $\begin{array}{c}\text { Transparent OPP } \\
\text { (Eureka) }\end{array}$ & $27.5 \pm 2.7$ & $33.9 \pm 2.0$ & $33.2 \pm 1.8$ & $30.3 \pm 2.6$ & $36.0 \pm 2.6$ & $32.2 \pm 2.7$ \\
\hline
\end{tabular}

Table 1 Surface roughness analysis on the six PSAs investigated 


\section{Figure captions}

Fig. 1. (a) Sketch of a force spectroscopy experiment and explanation of a typical force spectroscopy curve; (b) An $F$ - $d$ curve. The meanings of $F_{\max }, d_{\max }$, and $\gamma$ are identified.

Fig. 2. AFM phase images $5 \times 5 \mu \mathrm{m}^{2}$ and high-resolution zoomed AFM images $2 \times 2$ $\mu \mathrm{m}^{2}$ of the zones marked in squares for (a): transparent cello tape (the phase degree is $78-83^{\circ}$ for the $5 \times 5 \mu \mathrm{m}^{2}$ image and $80-88^{\circ}$ for the $2 \times 2 \mu \mathrm{m}^{2}$ image), (b): brown packaging tape (the phase degree is $81-89^{\circ}$ for the $5 \times 5 \mu \mathrm{m}^{2}$ image and $83-88^{\circ}$ for the $2 \times 2 \mu \mathrm{m}^{2}$ image) and (c): electrical insulation tape (the phase degree is $76-83^{\circ}$ for the $5 \times 5 \mu \mathrm{m}^{2}$ image and $80-85^{\circ}$ for the $2 \times 2 \mu \mathrm{m}^{2}$ image) .

Fig. 3. AFM phase images $5 \times 5 \mu \mathrm{m}^{2}$ and high-resolution zoomed AFM images $2 \times 2$ $\mu \mathrm{m}^{2}$ of the zones marked in squares for (a): OPP transparent cello tape by Henkel (the phase degree is $80-81^{\circ}$ for the $5 \times 5 \mu \mathrm{m}^{2}$ image and $80-82^{\circ}$ for the $2 \times 2 \mu \mathrm{m}^{2}$ image), (b) : OPP transparent cello tape by Niceday (the phase degree is $73-84^{\circ}$ for the $5 \times 5$ $\mu \mathrm{m}^{2}$ image and $77-85^{\circ}$ for the $2 \times 2 \mu \mathrm{m}^{2}$ image) and (c): OPP transparent cello tape by Eureka (the phase degree is $81-84^{\circ}$ for the $5 \times 5 \mu \mathrm{m}^{2}$ image and $80-85^{\circ}$ for the $2 \times$ $2 \mu \mathrm{m}^{2}$ image).

Fig. 4. Typical trace and retrace $F$ - $d$ curves obtained on (a): transparent cello tape, (b): brown packaging tape, and (c): electrical insulation tape.

Fig. 5. Histograms showing the statistical distribution of $F_{\max }, d_{\max }$, and $\gamma$ over the $F$ $d$ curves obtained at 400 different grid points distributed evenly on a $5 \times 5 \mu \mathrm{m}^{2}$ area of the adhesive in (a): transparent cello, (b): brown packaging, and (c): electrical insulation tapes.

Fig. 6. The 2 D AFM-FM adhesion energy maps on a grid of $20 \times 20$ points covering a $5 \times 5 \mu \mathrm{m}^{2}$ area of the adhesive in (a): the transparent cello tape, (b): the brown packaging tape, and (c): the electrical insulation tape. The brighter (darker) is the 
square, the higher (lower) the adhesion energy, and thus the softer (harder) is the component of the adhesive film. The grey-coloured chart scale on the right-hand side of each figure indicates the associated adhesion energy value in $10^{-15} \mathrm{~J}$ corresponding to each colour shade. The adhesion energy values vary in the range (a) $0-6220 \times 10^{-15}$ $\mathrm{J}$ for the transparent cello tape, (b) $0-390 \times 10^{-15} \mathrm{~J}$ for the brown tape; and (c) 0 $1025 \times 10^{-15} \mathrm{~J}$ for the electrical insulation tape.

Fig. 7. Typical trace and retrace $F-d$ curves obtained on (a): OPP transparent cello tape by Niceday, (b): OPP transparent cello tape by Henkel, and (c): OPP transparent cello tape by Eureka.

Fig. 8. Histograms showing the statistical distribution of $F_{\max }, d_{\max }$, and $\gamma$ over the $F$ $d$ curves obtained at 400 different grid points distributed evenly on a $5 \times 5 \mu \mathrm{m}^{2}$ area of the adhesive in (a): OPP transparent cello tape by Niceday, (b): OPP transparent cello tape by Henkel, and (c): OPP transparent cello tape by Eureka.

Fig. 9. The 2 D AFM-FM adhesion energy maps on a grid of $20 \times 20$ points covering a $5 \times 5 \mu \mathrm{m}^{2}$ area of the adhesive in (a): OPP transparent cello tape by Niceday, (b): OPP transparent cello tape by Henkel, and (c): OPP transparent cello tape by Eureka. The brighter (darker) is the square, the higher (lower) the adhesion energy, and thus the softer (harder) is the component of the adhesive film. The grey-coloured chart scale on the right-hand side of each figure indicates the associated adhesion energy value in $10^{-15} \mathrm{~J}$ corresponding to each colour shade. The scale is $0-1200 \times 10^{-15} \mathrm{~J}$ for each map. 


\section{Figures}

(a)

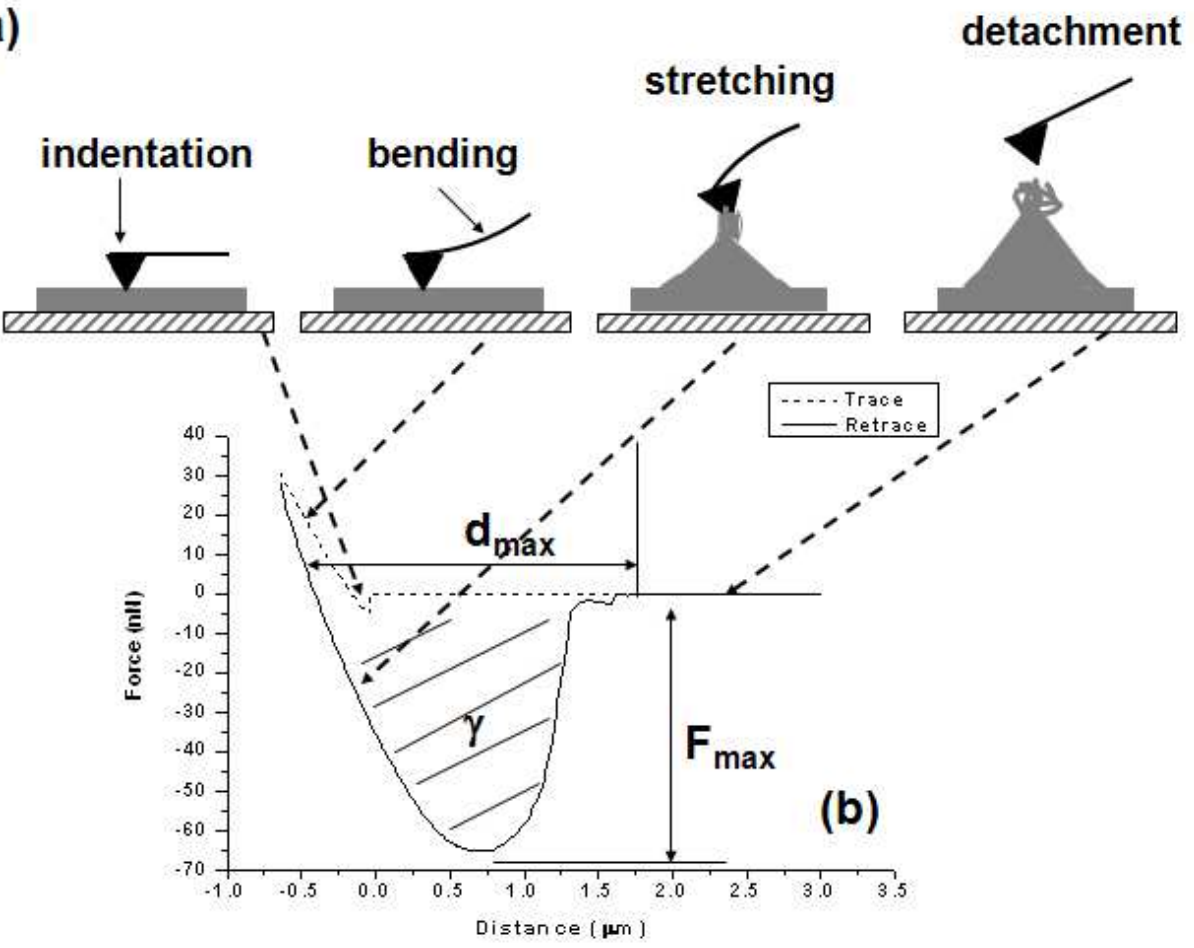

Figure 1 

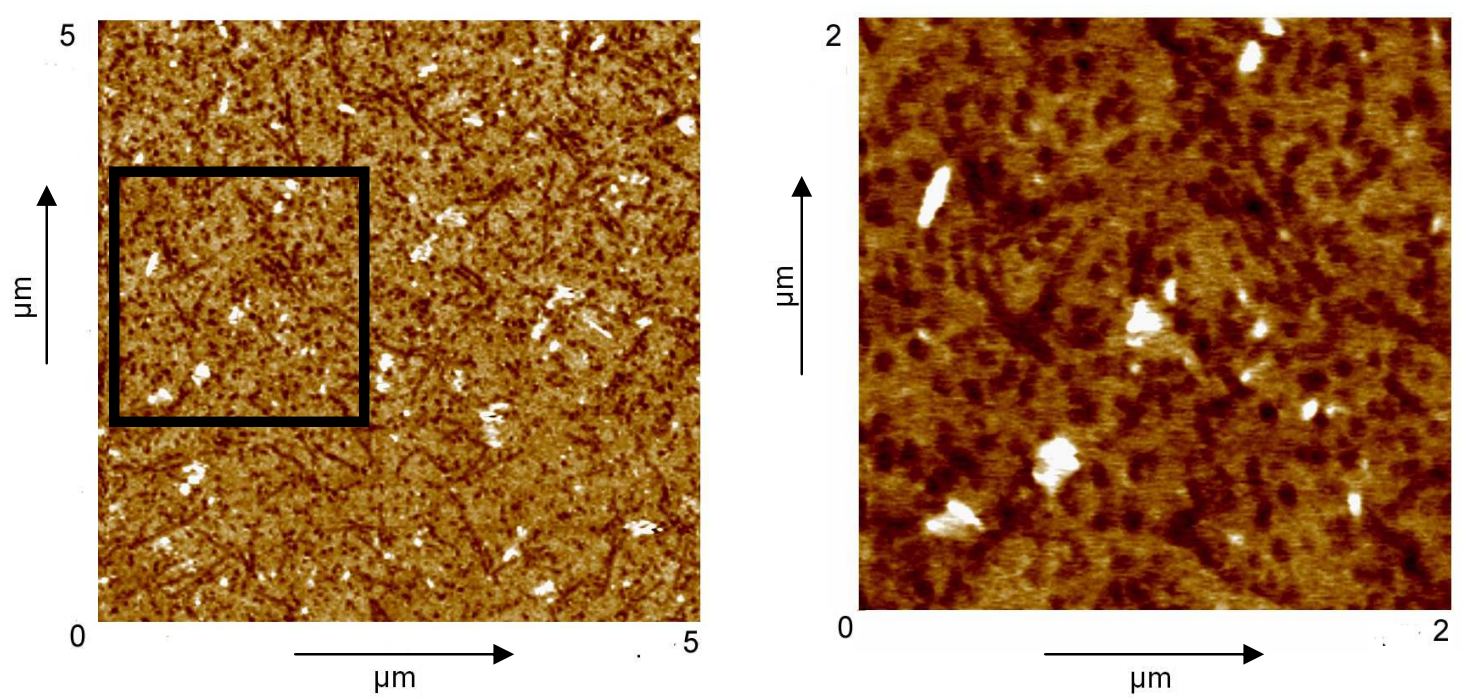

(a) : Transparent cello tape
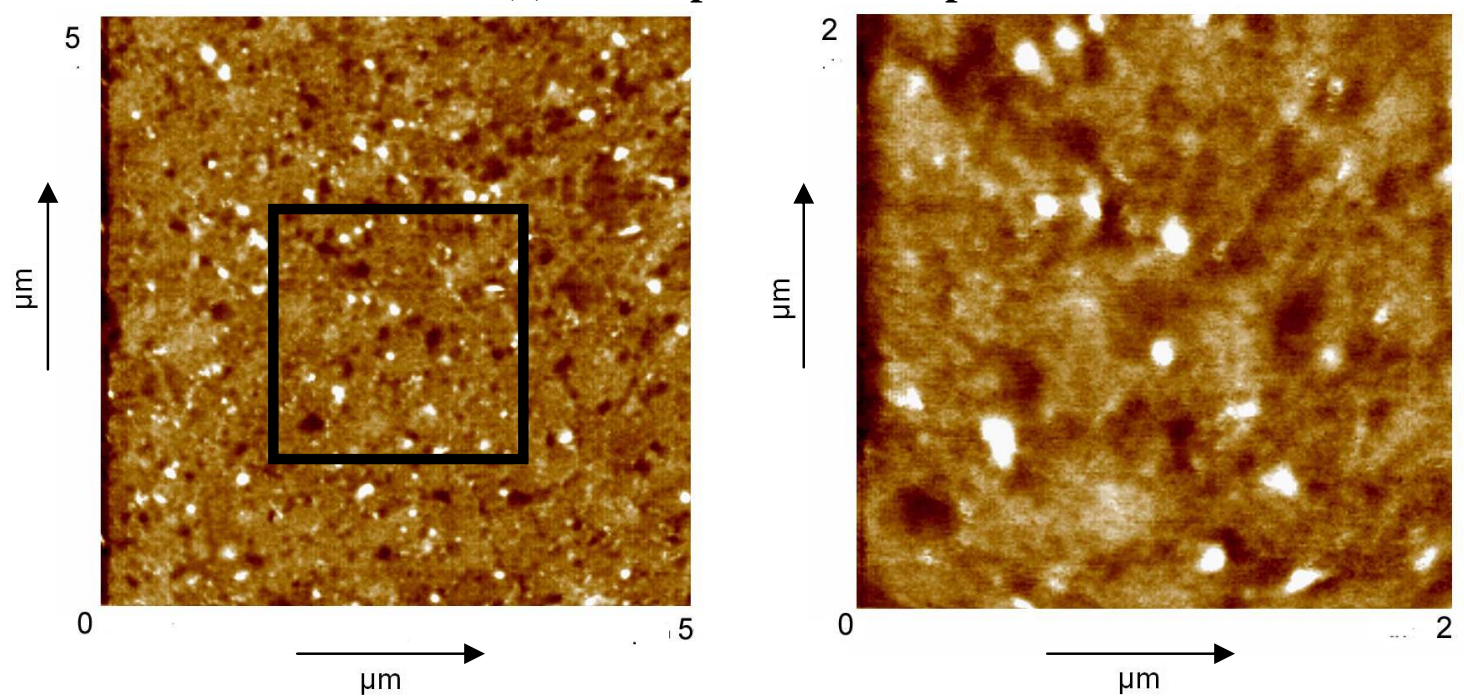

(b) : Brown packaging tape
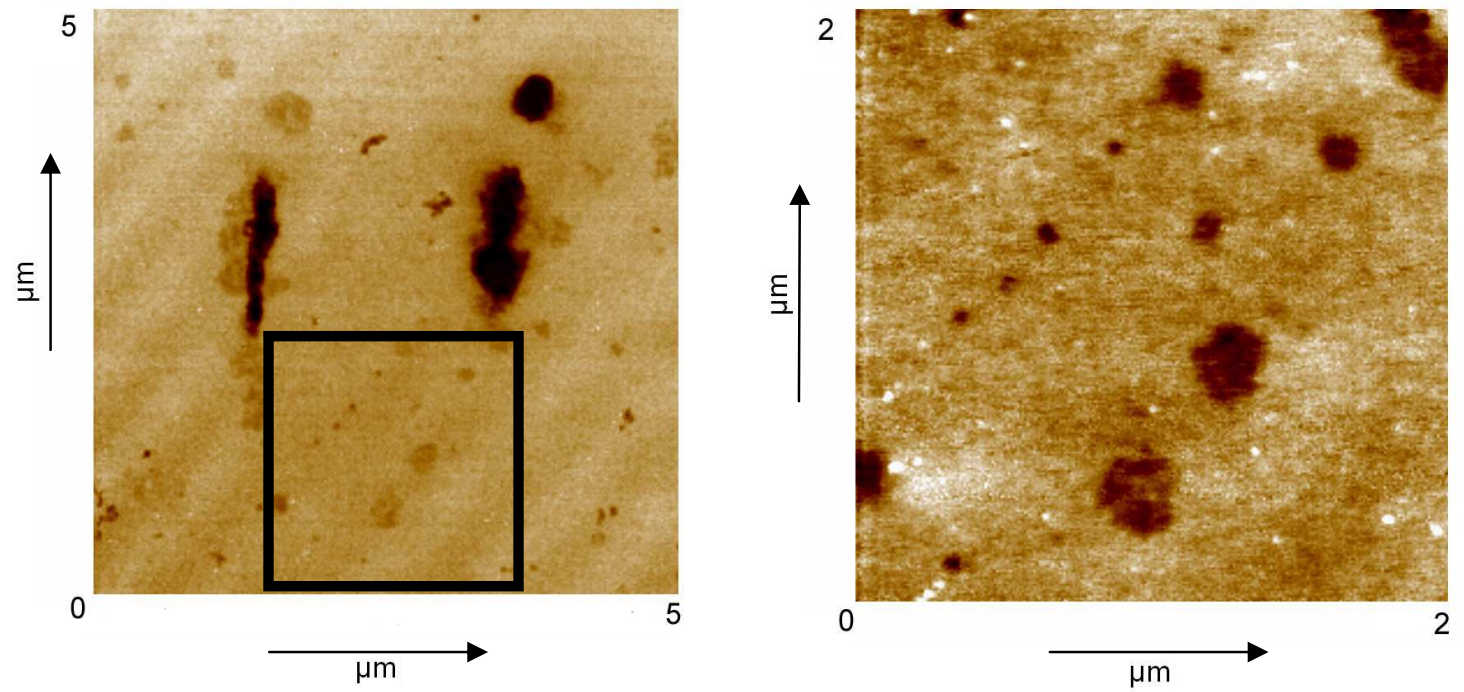

(c) : Electrical insulation tape

Figure 2 

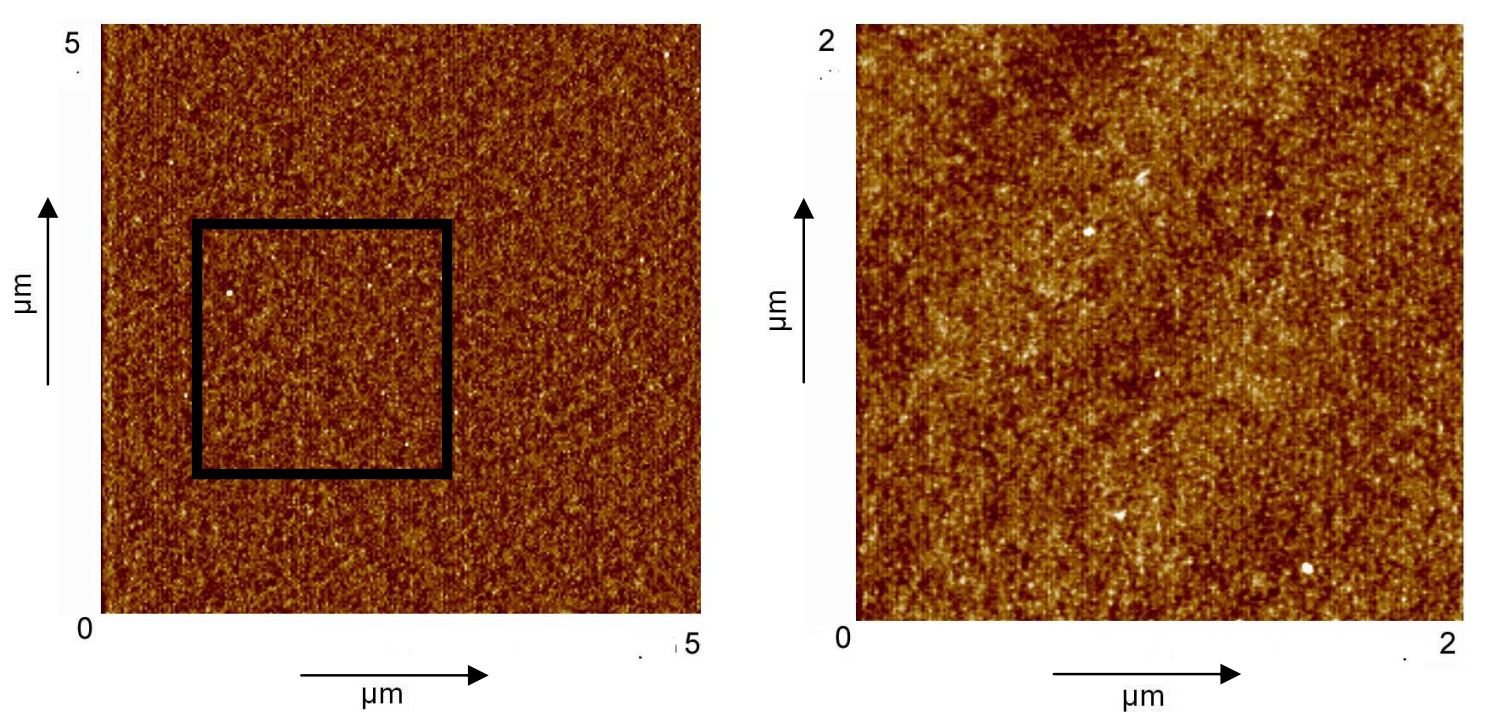

(a) : OPP transparent - Henkel
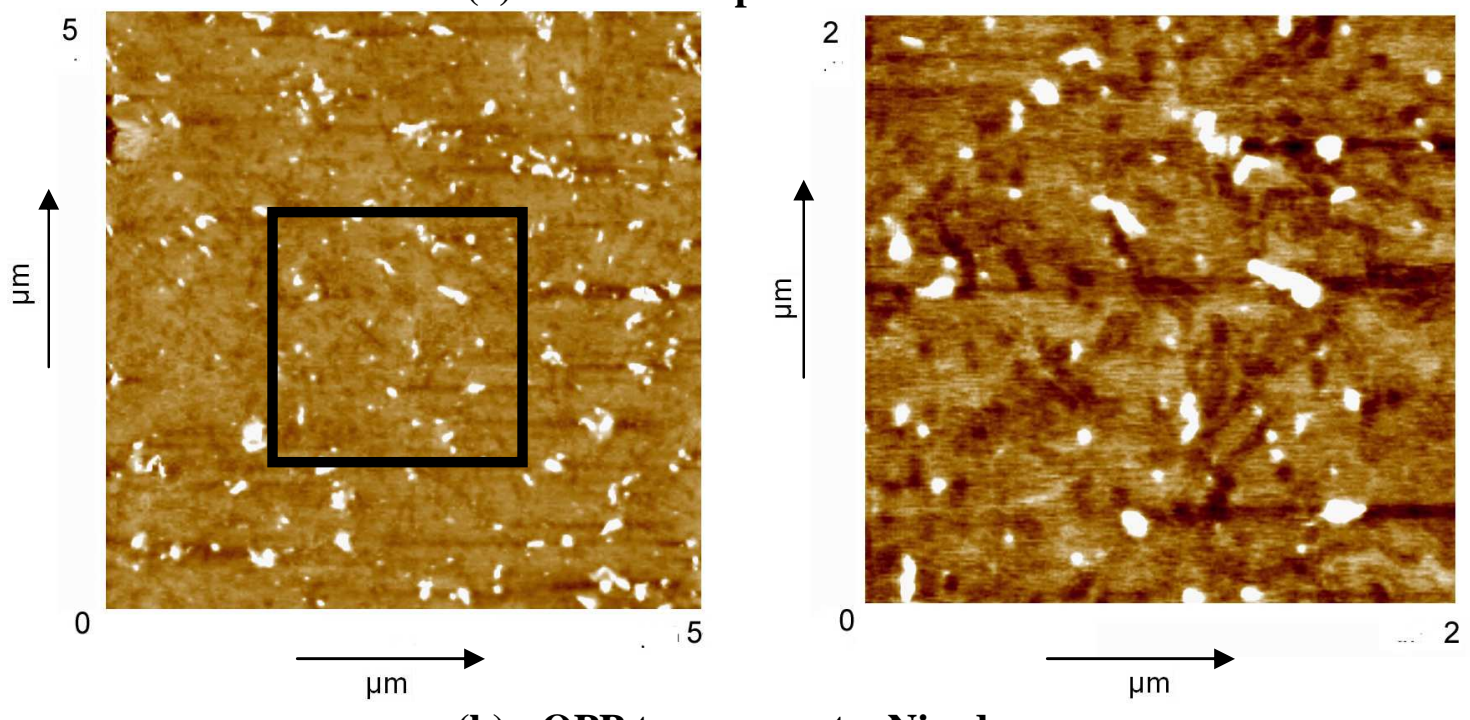

(b) : OPP transparent - Niceday
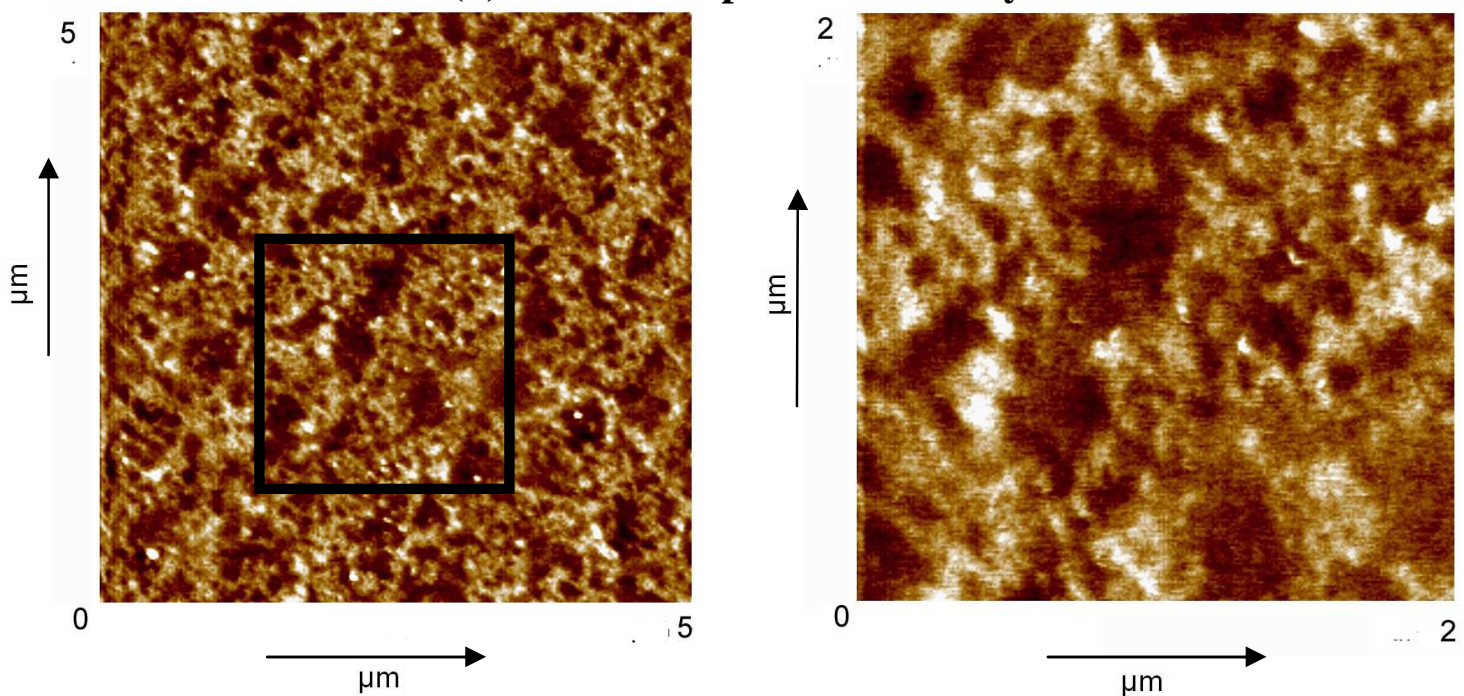

(c) : OPP transparent - Eureka

Figure 3 


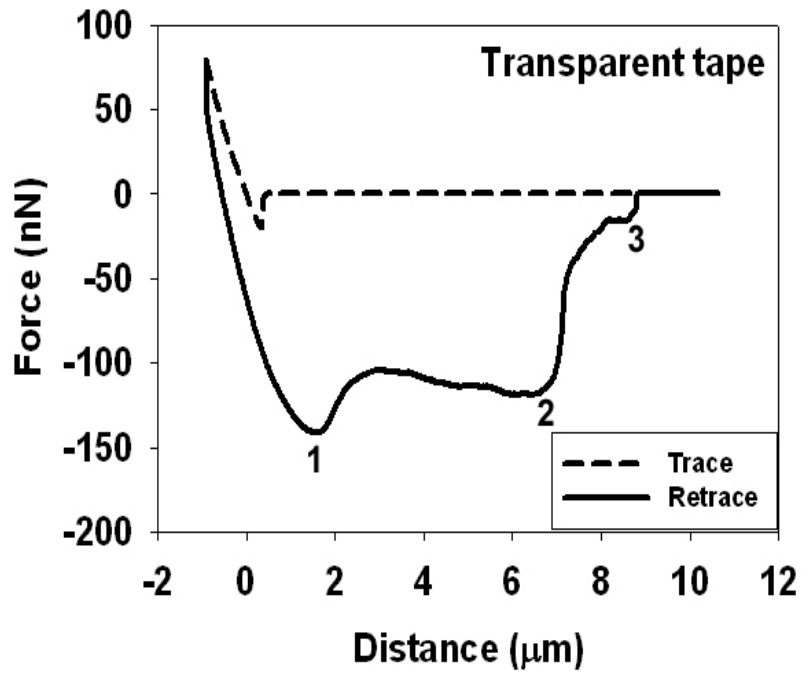

(a)

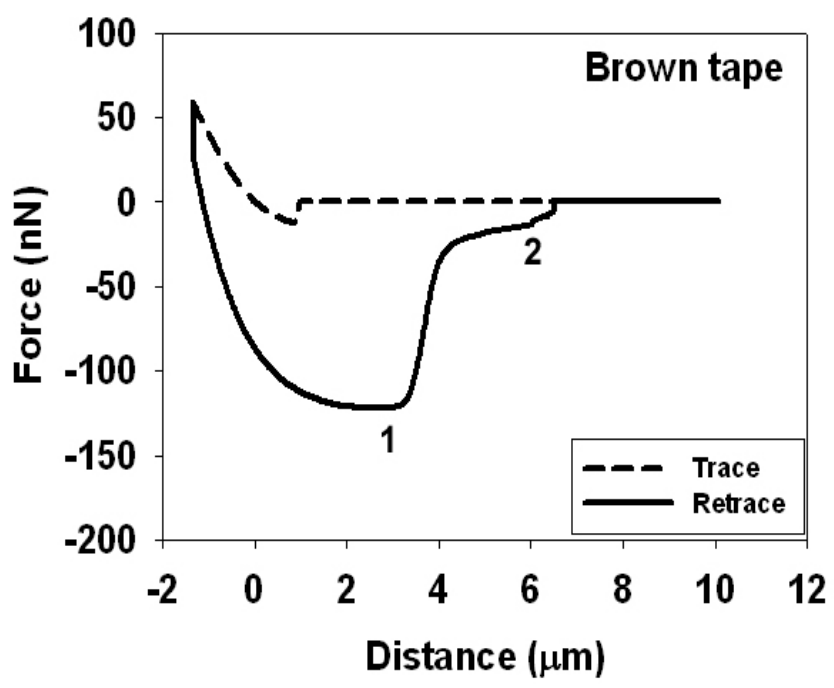

(b)
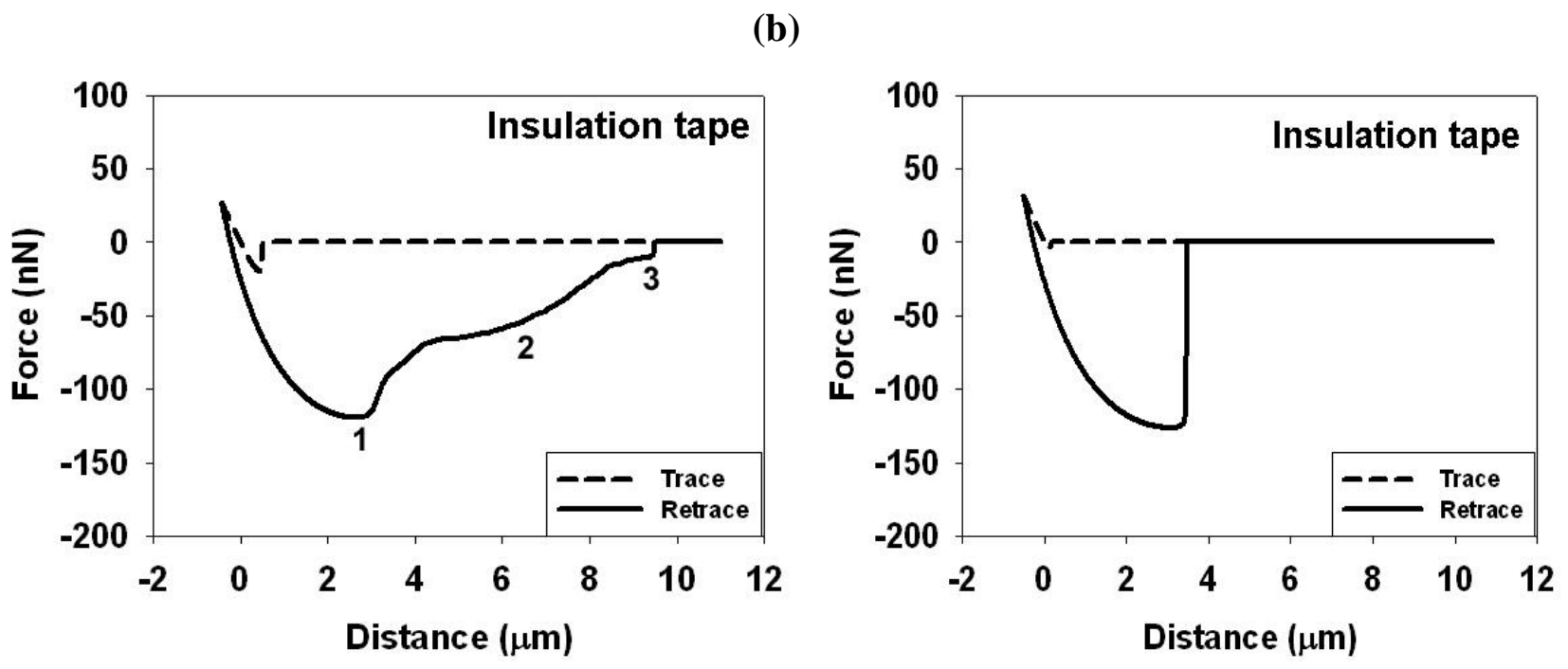

(c)

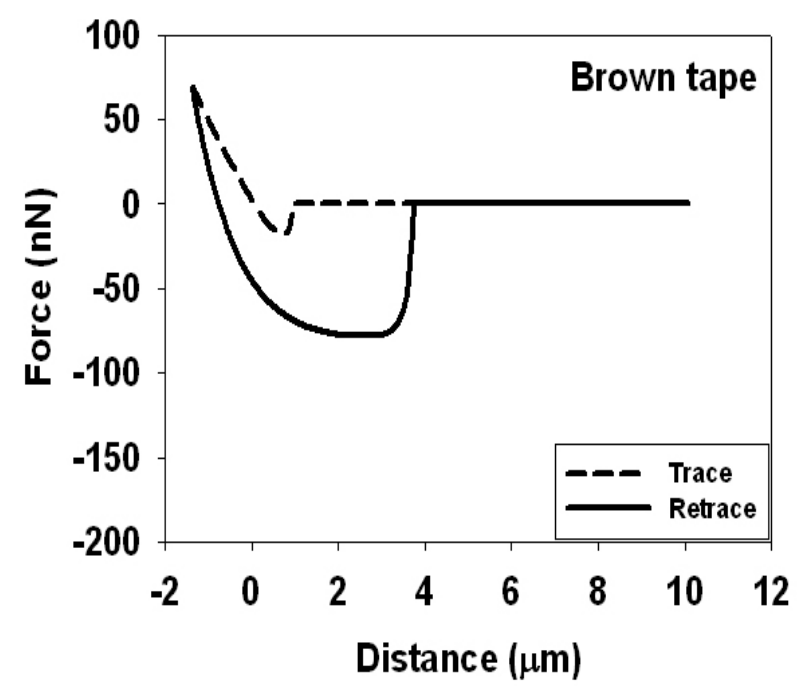

Figure 4 

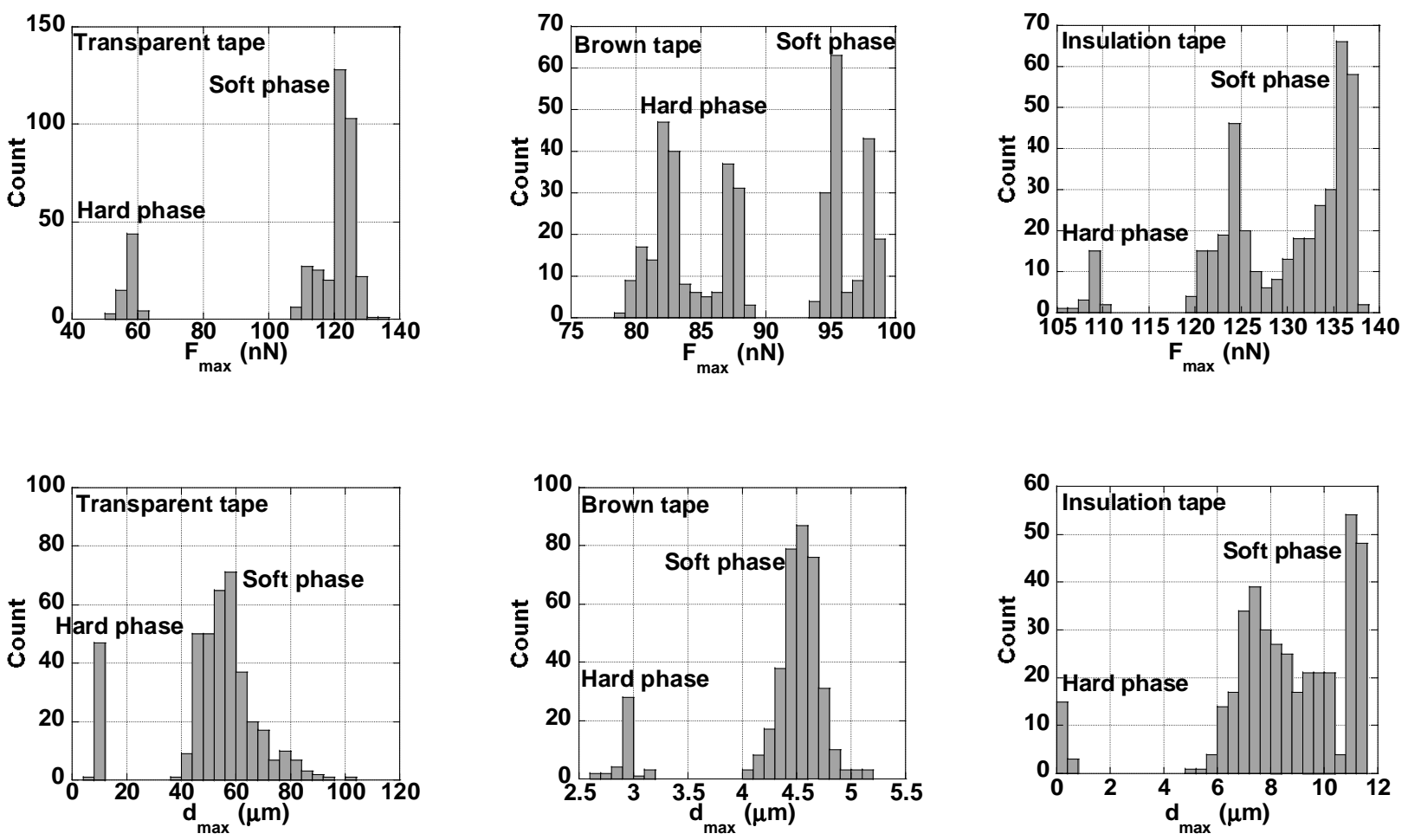

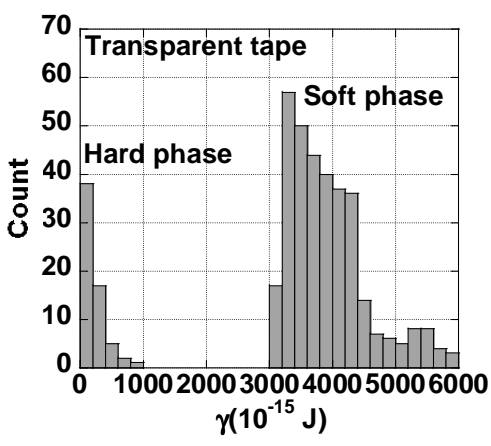

(a)

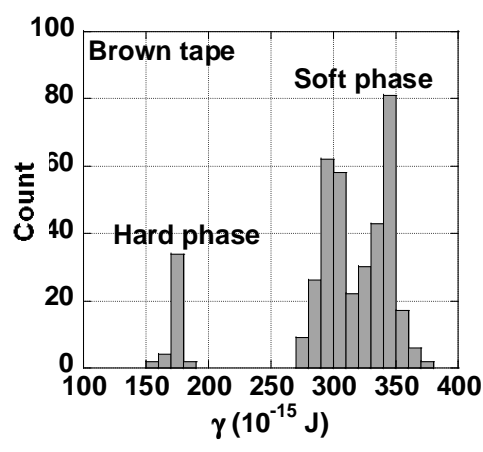

(b)

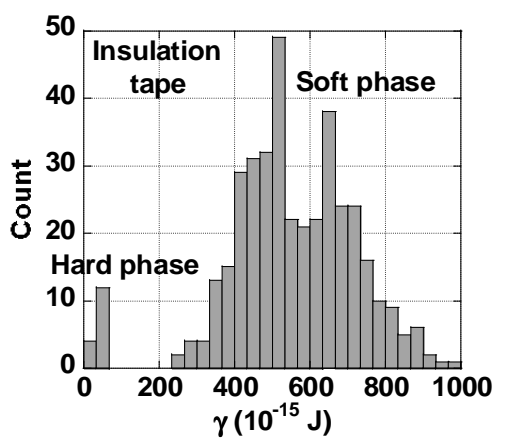

(c)

Figure 5 

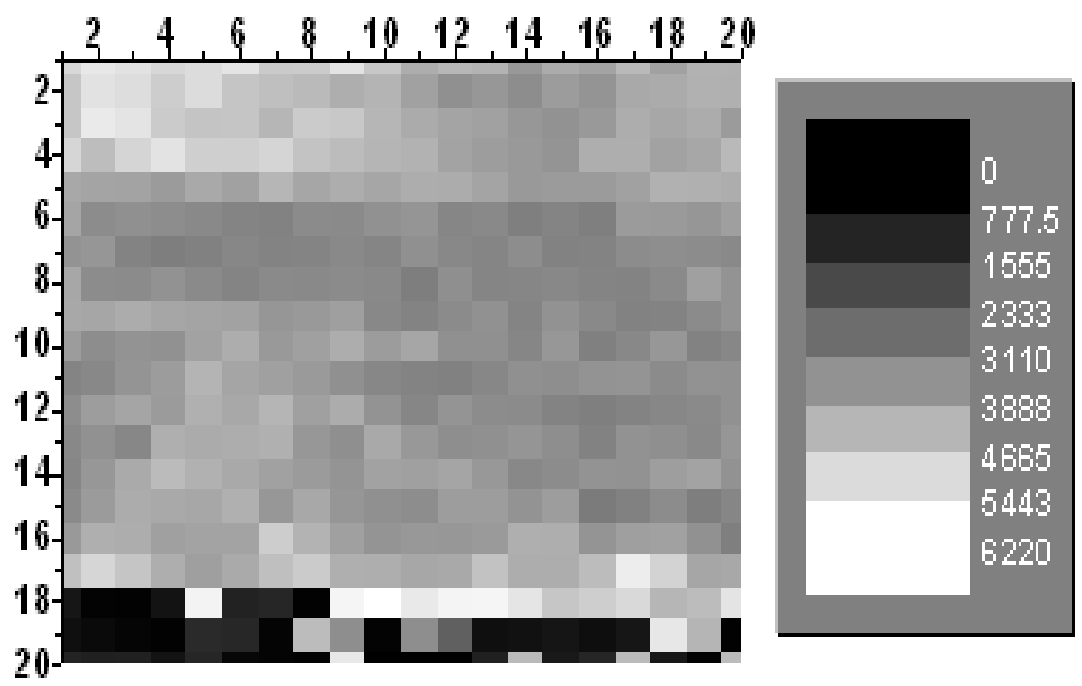

(a): Transparent cello tape

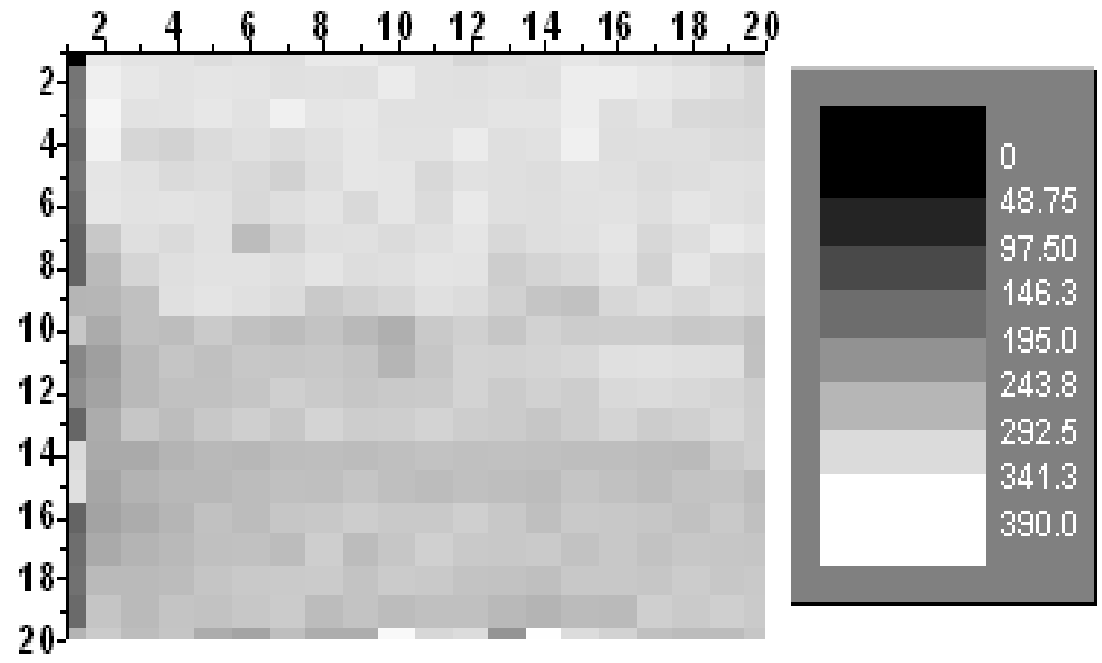

(b): Brown packaging tape

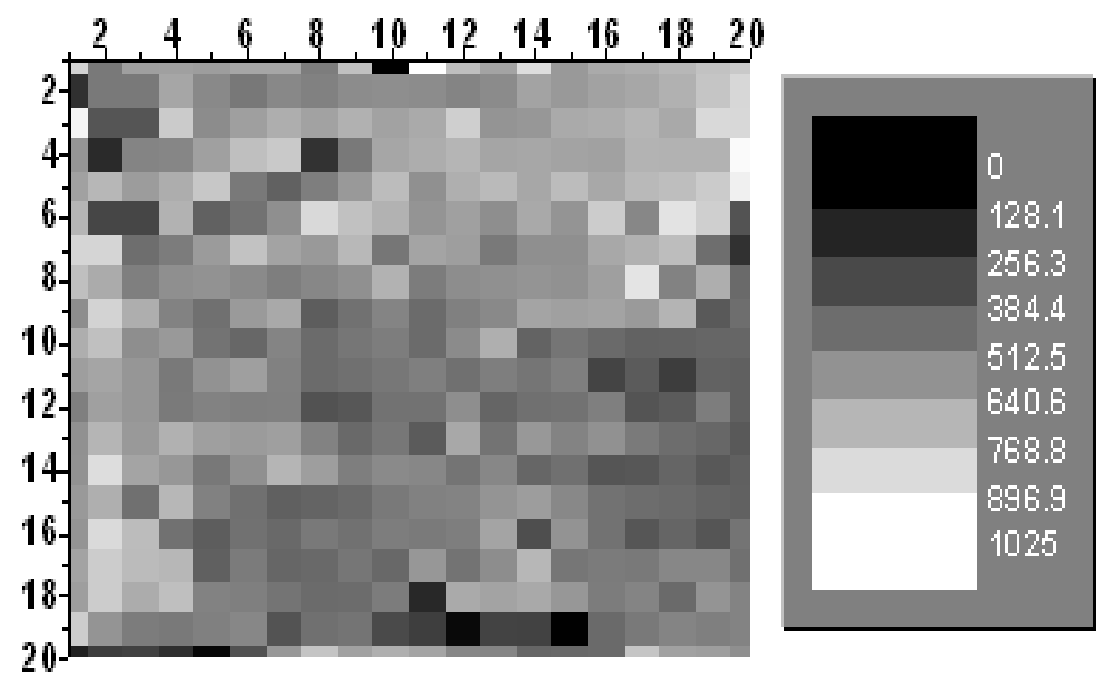

(c) : Electrical insulation tape

Figure 6 

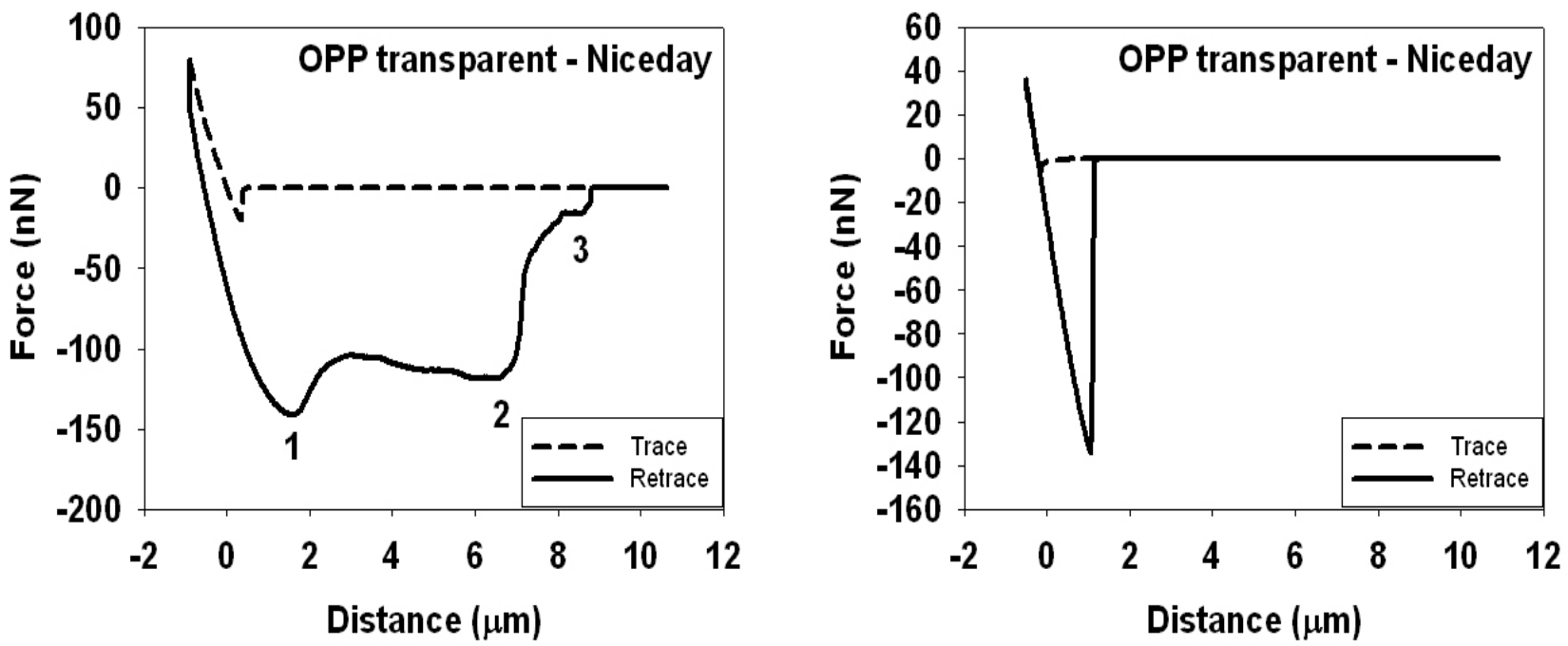

(a)

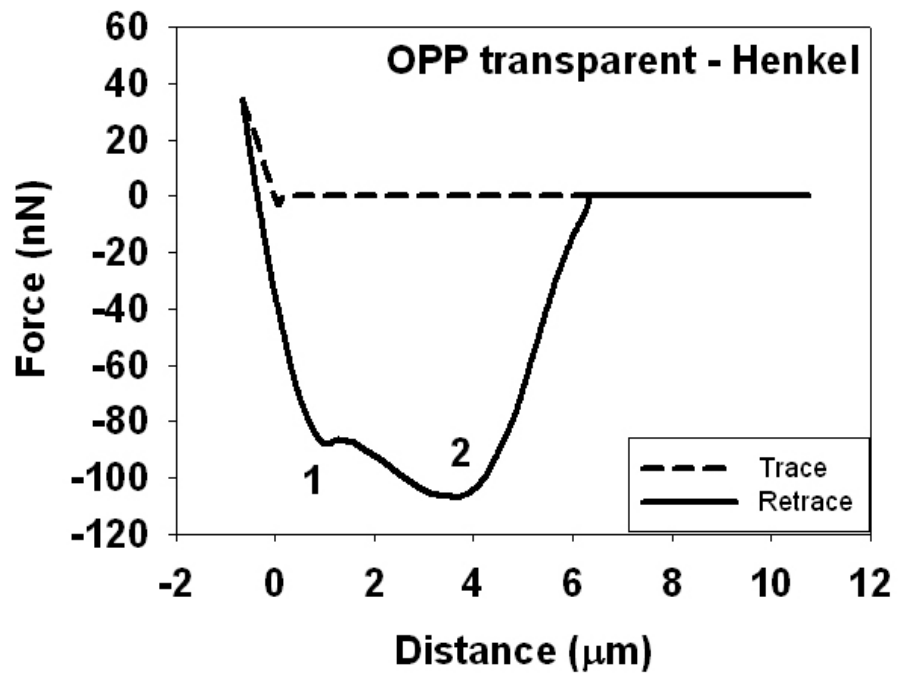

(b)
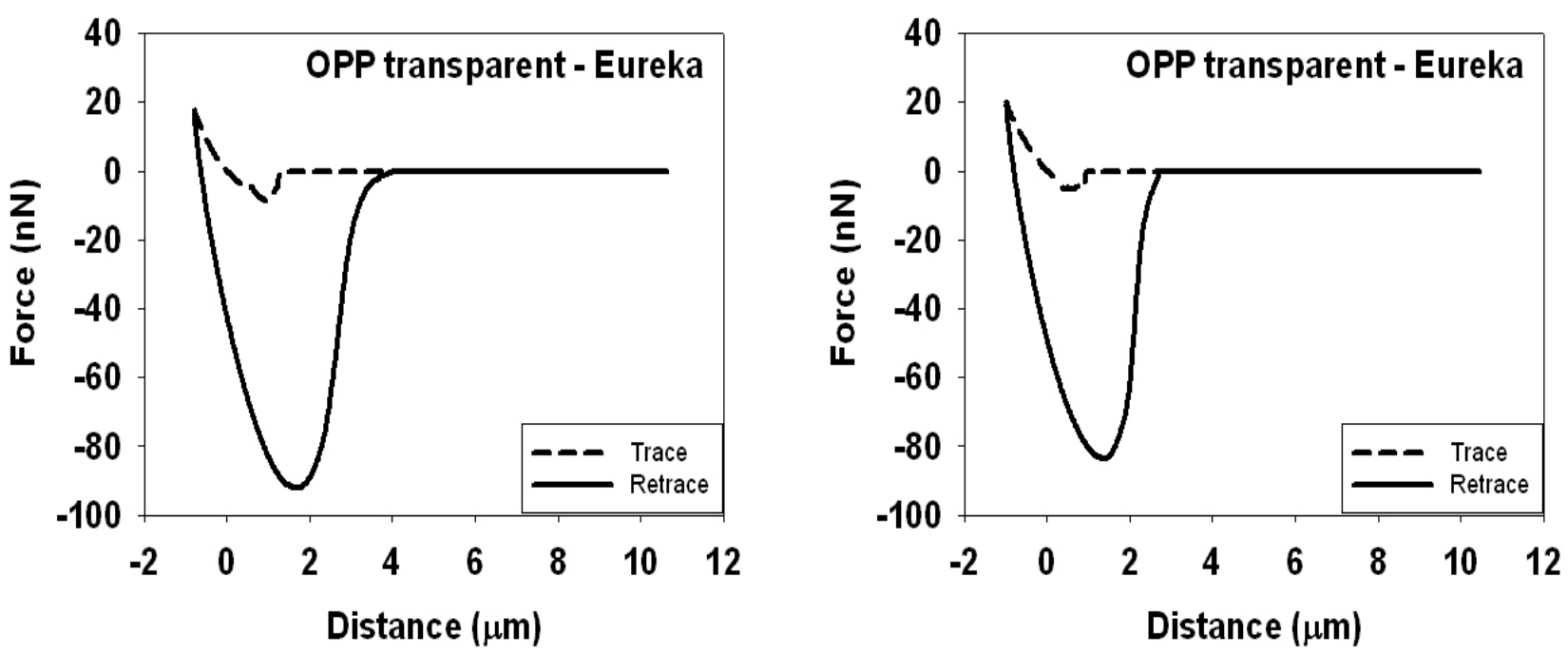

(c)

Figure 7 



(a)

(b)

(c)

Figure 8 

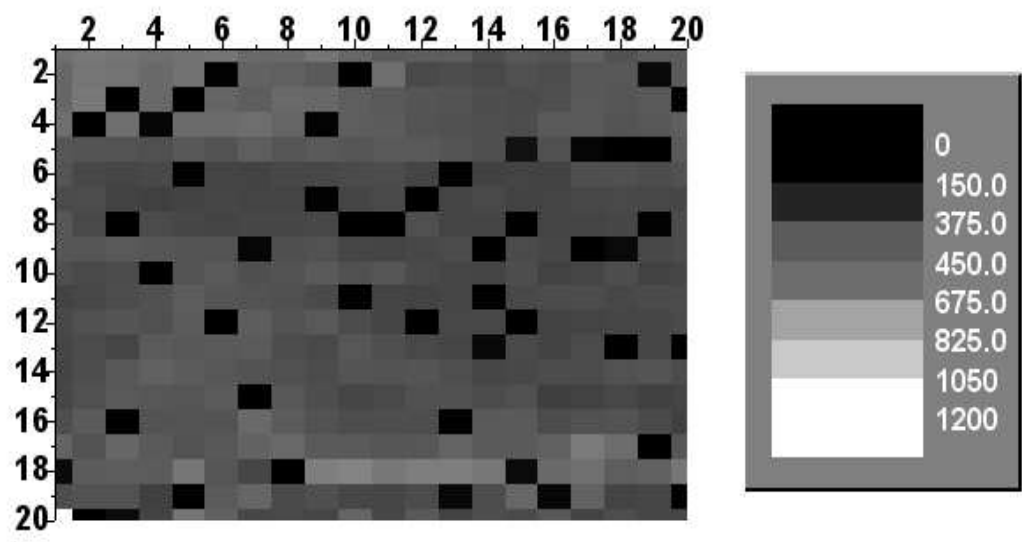

(a): OPP transparent - Niceday
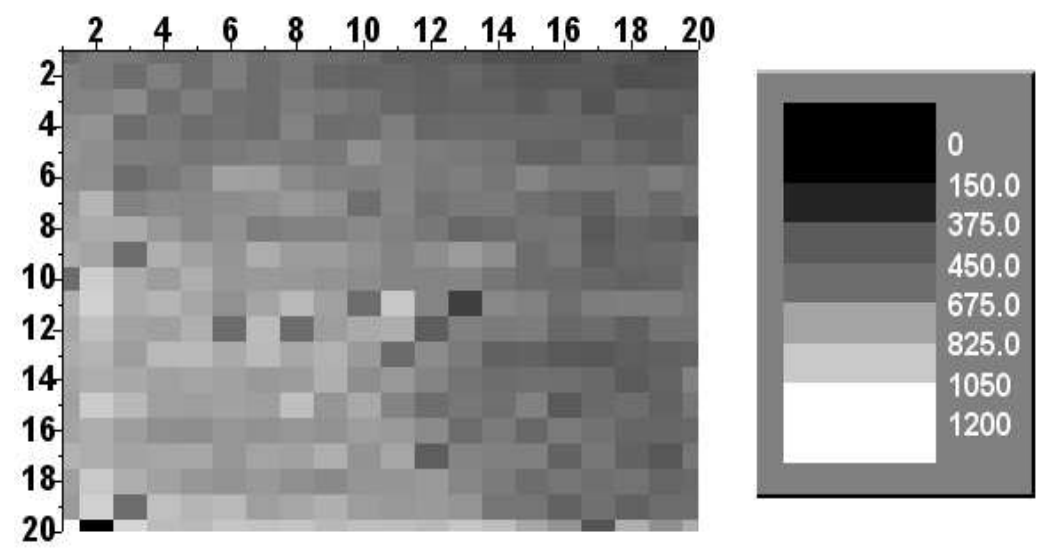

(b): OPP transparent - Henkel
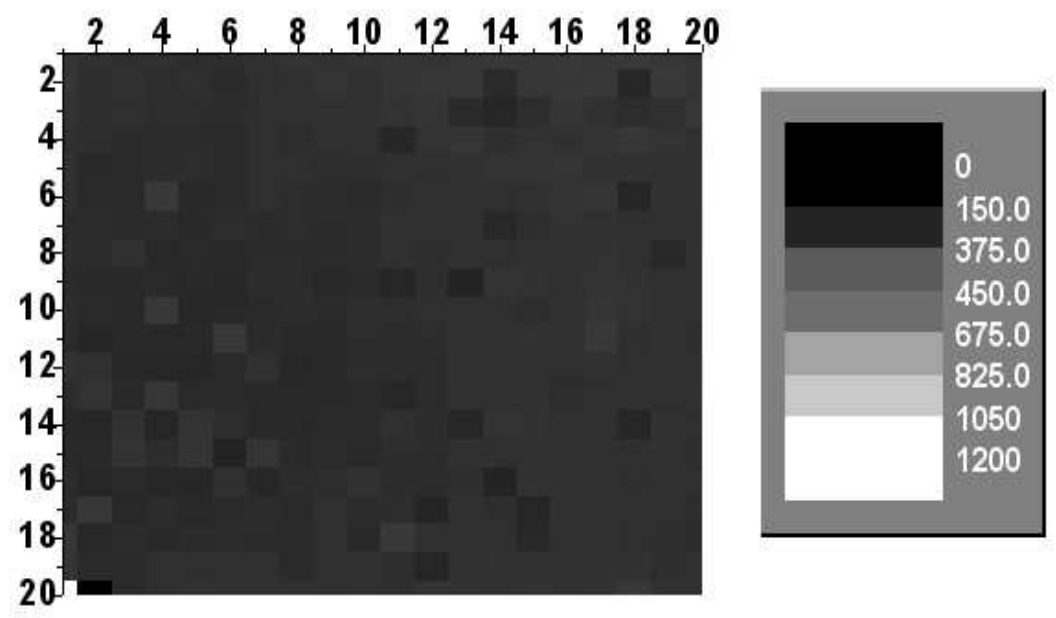

(c) : OPP transparent - Eureka

Figure 9 


\section{References}

[1] G. Payne, C. Wallace, B. Reedy, et al., Visible and near-infrared chemical imaging methods for the analysis of selected forensic samples, Talanta 67 (2005) 334-344.

[2] G. Binning, C.F. Quate, C. Gerber, Atomic Force Microscopy, Phys. Rev. Lett. 56 (1986) 930-933.

[3] H. Kitano, A.M. Niwa, S. Fujinami, et al., Young's modulus mapping on hair cross-section by atomic force microscopy, Composite Interfaces, 16 (2009) 1-12.

[4] Y. Chen, J.Y. Cai, Membrane deformation of unfixed erythrocytes in air with time lapse investigated by tapping mode atomic force microscopy, Micron, 37 (2006) 339-346.

[5] S. Kasas, A. Khanmy-Vital, G. Dietler, Examination of line crossings by atomic force microscopy, Forensic Science International, 119 (2001) 290-298.

[6] H. Zhang, R.N. Lamb, Superhydrophobic treatment for textiles via engineering nanotextured silica/polysiloxane hybrid material onto fibres, Surface Engineering, 25 (2009) 21-24.

[7] E. Canetta, A.K. Adya, Morphological changes in textile fibres exposed to environmental stresses: Atomic force microscopic examination, Forensic Science International, 191 (2009) 6-14.

[8] G. Tosello, A. Gava, H.N. Hansen, G. Lucchetta, F. Marinello, Characterisation and analysis of weld lines on micro injection moulded parts using atomic force microscopy, Wear 266 (2009) 534-538.

[9] E. Canetta, J. Marchal, C.H. Lei, et al., A comparison of tackified, miniemulsion core-shell acrylic latex films with corresponding particle-blend films: structureproperty relationship, Langmuir 25 (2009) 11021-11031.

[10] T. Wang, E. Canetta, T.G. Weerakkody, J.L. Keddie, U. Rivas, pH dependence of the properties of waterborne pressure-sensitive adhesives containing acrylic acid, Applied Materials and Interfaces 1 (2009) 631-639.

[11] P. Maynard, K. Gates, C. Roux, C. Lennard, Adhesive tape analysis: Establishing the evidential value of specific techniques, Journal of Forensic Sciences 46 (2001) 280-287.

[12] Y. Kumooka, Analysis of deteriorated rubber-based pressure sensitive adhesive by pyrolysis-gas chromatograph/mass spectrometry and attenuated total reflectance Fourier transform infrared spectrometry, Forensic Science International 163 (2006) 132-137.

[13] Y. Kumooka, Analysis of rosin and modified rosin esters in adhesives by matrixassisted laser desorption/ionization time-of-flight mass spectrometry (MALDITOF-MS), Forensic Science International 176 (2008) 111-120.

[14] J.V. Goodpaster, A.B. Sturdevant, K.L. Andrews, E.M. Briley, L. Brun-Conti, Identification and comparison of electrical tapes using instrumental and 
statistical techniques: II. Organic composition of the tape backing abd adhesive, J. Forensic Sci. 54 (2009) 328-338.

[15] Y. Kumooka, Hierarchical cluster analysis as a tool for preliminary discrimination of ATR-FT-IR of OPP acrylic and rubber-based adhesives, Forensic Science International 189 (2009) 104-110.

[16] Y. Kumooka, Classification of OPP adhesive tapes according to MALDI mass spectra of adhesives, Forensic Science International 197 (2010) 75-79.

[17] J. Huttunen, C. Austin, M. Dawson, C. Roux, J. Robertson, Physical evidence in drug intelligence, Part 1: Rationale based on hierarchic distribution of drugs using pyrolysis gas chromatography -mass spectrometry as an example, Australian Journal of Forensic Sciences 39 (2007) 93 - 106.

[18] J. Huttunen, P. Doble, M. Dawson, C. Roux, J. Robertson, Physical evidence in drug intelligence, Part 2: Discrimination of packaging tapes by colour, Australian Journal of Forensic Sciences, 40 (2008) 73-83.

[19] Scientific Working Group for Materials Analysis (SWGMAT). Standards and Guidelines for the Forensic Examination of Pressure-Sensitive Tapes. Forensic Science Communications, 10 (4) (2008). http://www2.fbi.gov/hq/lab/fsc/backissu/oct2008/standards/2008_10_standards0 2.htm

[20] J. Mallégol, O. Dupont, J.L. Keddie, Obtaining and interpreting images of acrylic pressure sensitive adhesives by tapping mode atomic force microscopy, Langmuir 17 (2001) 7022-7031.

[21] C.H. Lei, K. Ouzineb, O. Dupont, J. L. Keddie, Probing Particle Structure in Waterborne Pressure-Sensitive Adhesives with Atomic Force Microscopy, J. Coll. Interf. Sci. 307 (2007) 56-63.

[22] W.W. Scott, B. Bhushan, Use of phase imaging in atomic force microscopy for measurement of viscoelastic contrast in polymer nanocomposites and molecularly thick lubricant films, Ultramicroscopy 97 (2003) 151-169.

[23] B. Anczykowski, B. Gotsmann, H. Fuchs, J.P. Cleveland, V.B. Elings, How to measure energy dissipation in dynamic mode atomic force microscopy, Appl. Surf. Sci. 140 (1999) 376-382.

[24] A. Pavia, N. Sheller, M.D. Foster, A. J. Crosby, K. R. Shull, Study of the surface adhesion of pressure-sensitive adhesives by atomic force microscopy and spherical indenter tests, Macromolecules 33 (2000) 1878-1881.

[25] A. Pavia, N. Sheller, M.D. Foster, A.J. Crosby, K.R. Shull, Microindentation and nanoindentation studies of aging in pressure-sensitive adhesives, Macromolecules 34 (2001) 2269-2276.

[26] S. Moon, M.D. Foster, Influence of Humidity On Surface Behavior Of Pressure Sensitive Adhesives Studied Using Scanning Probe Microscopy, Langmuir 18 (2002) 8108-8115. 
[27] F. Benmouna, T.D. Dimitrova, D. Johannsmann, Nanoscale Mapping of the Mechanical Properties of Polymer Surfaces by Means of AFM Noise Analysis: Spatially Resolved Fibrillation of Latex Films, Langmuir 19 (2003) 1024710253.

[28] S. Moon, S. Swearingen, M.D. Foster, Study of Dynamic Adhesion Behavior using Atomic Force Microscopy, Polymer 45 (2004) 5951- 5959. 